University of Texas Rio Grande Valley

ScholarWorks @ UTRGV

Physics and Astronomy Faculty Publications and Presentations

$12-1-2013$

\title{
Detection of pulsar beams deflected by the black hole in SGR A*: Effects of black hole spin
}

Sourabh Nampalliwar

Richard H. Price

Teviet Creighton

Fredrick A. Jenet

Follow this and additional works at: https://scholarworks.utrgv.edu/pa_fac

Part of the Astrophysics and Astronomy Commons

\section{Recommended Citation}

Sourabh Nampalliwar, et. al., (2013) Detection of pulsar beams deflected by the black hole in SGR A*: Effects of black hole spin.Astrophysical Journal778:2. DOI: http://doi.org/10.1088/0004-637X/778/2/145

This Article is brought to you for free and open access by the College of Sciences at ScholarWorks @ UTRGV. It has been accepted for inclusion in Physics and Astronomy Faculty Publications and Presentations by an authorized administrator of ScholarWorks @ UTRGV. For more information, please contact justin.white@utrgv.edu, william.flores01@utrgv.edu. 


\title{
DETECTION OF PULSAR BEAMS DEFLECTED BY THE BLACK HOLE IN SGR A*: EFFECTS OF BLACK HOLE SPIN
}

\author{
Sourabh Nampalliwar ${ }^{1},{\text { Richard H. } \text { Price }^{2} \text {, Teviet Creighton }}^{2}$, and Fredrick A. Jenet ${ }^{2}$ \\ ${ }^{1}$ Department of Physics \& Astronomy and Center for Gravitational Wave Astronomy, \\ University of Texas at Brownsville, Brownsville, TX 78520, USA \\ ${ }^{2}$ Department of Physics \& Astronomy, Center for Gravitational Wave Astronomy and Center for Advanced Radio Astronomy, \\ University of Texas at Brownsville, Brownsville, TX 78520, USA \\ Received 2012 June 20; accepted 2013 September 26; published 2013 November 12
}

\begin{abstract}
Some Galactic models predict a significant population of radio pulsars close to the Galactic center. Beams from these pulsars could be strongly deflected by the supermassive black hole (SMBH) believed to reside at the Galactic center and as a result reach Earth. Earlier work assuming a Schwarzschild SMBH gave marginal chances of observing this exotic phenomenon with current telescopes and good chances with future telescopes. Here we study whether those estimates are significantly affected by SMBH spin. We find that spin effects make a negligible difference in detectability, but the pattern of pulse arrival times is clearly affected. In particular, if strongly deflected beams are detected, the SMBH spin signature could be extracted from pulsar beam times of arrival.
\end{abstract}

Key words: black hole physics - Galaxy: nucleus - pulsars: general

Online-only material: color figures

\section{INTRODUCTION}

It is believed that the center of essentially every large galaxy, including our own, contains a supermassive black hole (SMBH). Sagittarius $A^{*}$, first discovered as a bright radio source, is expected to host the SMBH at the center of the Milky Way. Observations of the region in the infrared (Genzel et al. 2003; Gillessen et al. 2009), radio (Doeleman et al. 2008; Broderick et al. 2009), X-ray (Baganoff et al. 2003; Porquet et al. 2003), and optical (Ghez et al. 2008) spectra, have revealed characteristics of the central object such as mass and accretion flow. Alternatives to an SMBH, such as a dense cluster of dark stellar objects or a ball of massive, degenerate fermions (Schödel et al. 2002) have largely been ruled out. These observations have also suggested an initial mass function (IMF) near the SMBH strongly tilted toward massive stars (Nayakshin \& Sunyaev 2005; Maness et al. 2007; Levin 2006). This top-heavy IMF suggests a large population of neutron stars orbiting the SMBH. Estimates place $\sim 10,000$ of them within $1 \mathrm{pc}$ of Sgr A* (Muno et al. 2005) and 1000 within 0.01 pc (Pfahl \& Loeb 2004). Beams from these pulsars could traverse the strong field region of the SMBH, be deflected, and subsequently reach Earth. Using the precision of pulsar timing, we might be able to detect these deflected beams and from them infer properties of the strong field region through which the beams have passed.

Previous work in this direction with the assumption of a non-spinning SMBH has been reported in Wang et al. (Wang et al. 2009a, 2009b) and Stovall et al. (Stovall et al. 2012, hereafter Paper I, II, and III, respectively). In Paper I, strong field effects on the intensity and times of arrival (TOAs) of pulsar beams were analyzed in the case where the pulsar beam is confined to the plane of the pulsar orbit. Paper II extended the formalism to general orientations. In Paper III, an estimate was given of the probability of observing these deflected pulsar beams. For the pulsar luminosity distribution calculated in the ATNF Pulsar Catalog, ${ }^{3}$ it was found

\footnotetext{
3 http://www.atnf.csiro.au/research/pulsar/psrcat/
}

that there is a marginal probability of detection with current instruments like the Parkes Radio Telescope and the Green Bank Telescope, and future instruments, in particular FAST and the Square Kilometre Array, provide a reasonable chance of detection. For a more conservative distribution (FaucherGigurè \& Kaspi 2006), chances of detection with existing telescopes are weak, but detection with future instruments remains promising.

Paper III was based on the model of Paper II, and hence embodied the assumption of a non-spinning SMBH. The Sgr A* SMBH is certainly expected to be rotating, though the rate of rotation is indeterminate, with predictions for the spin rate ranging from very slow (Liu \& Melia 2002) to moderate (Genzel et al. 2003). In principle, spin effects could have a dramatic effect on beam bending and hence on the probability of the detection of deflected beams. The current paper deals with an omission from earlier studies: the role of SMBH spin. The focus will be on the effect of spin on detectability to determine whether the detection probability estimates obtained for a non-rotating SMBH are significantly different and especially whether they might improve the prospects for near-term detection. A second purpose of this paper is to check whether the SMBH spin would have a strong enough effect on pulsar timing that spin information could be extracted from the arrival times of the pulses in a deflected beam.

The paper is organized as follows. In Section 2, we give an overview of the previous work and redefine terms that will be useful for purposes of generalization and comparison. We compare deflection of beams by a rotating SMBH and that by a non-rotating SMBH in Section 3. Section 4 describes the geometry of the system: beams emitted from a pulsar revolving around a spinning SMBH. This section also outlines the scheme for calculating probabilities for a detection. Section 5 compares the range of detection of deflected beams in a pulsar/ spinning-SMBH system to that for a pulsar/SchwarzschildSMBH system. Section 6 compares the pattern of pulse TOAs in the two scenarios. We conclude in Section 7. Throughout the paper, we will use the natural units $(c=1, G=1)$ and describe distances and time in terms of the black hole mass $M$. 


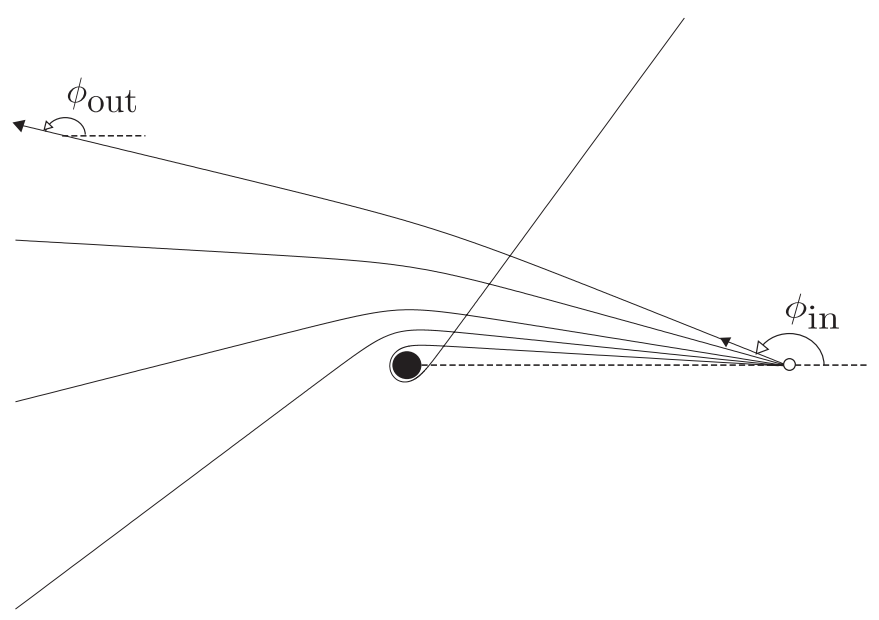

Figure 1. Trajectories of photons passing through the strong field region of a Schwarzschild black hole, emitted by a pulsar far away from the strong field region. Angles $\phi_{\text {in }}$ and $\phi_{\text {out }}$ are illustrated for one of the trajectories. This figure also serves for photons emitted in the equatorial plane of a spinning black hole.

\section{REVIEW OF THE SCHWARZSCHILD CASE}

As observed in Paper III, for a pulsar revolving around a Schwarzschild SMBH, there is a "region of detectability"-a range of Earth angular positions on the pulsar sky at which both direct and deflected beams are detectable. We start by reviewing the determination of this region.

Figure 1 sketches the trajectory of photons outside a Schwarzschild black hole. Also illustrated is the set of angles we use to describe a particular trajectory. Here $\phi_{\text {in }}$ corresponds to the angle at which the photon is emitted relative to the radially outward direction at the point of emission in a spherical coordinate system centered at the $\mathrm{SMBH} ; \phi_{\text {out }}$ corresponds to the angle measured relative to the same radial direction far away from the strong field region of the SMBH. Paper I relates these two angles by a function $F$ :

$$
\phi_{\text {out }}=F\left(\phi_{\text {in }} ; r_{0}\right)
$$

where $r_{0}$ is the pulsar-SMBH distance at the time of emission. In the absence of deflection, $\phi_{\text {out }}$ will be equal to $\phi_{\text {in }}$.
Paper III notes that deflection will change the beam intensity. There is a narrow range of $\phi_{\text {out }}$ around $\phi_{\text {out }}=\phi_{\text {out } \mid \text { peak }}=\pi$ for which there is dramatic amplification corresponding to strong lensing, but in general strongly bent beams will be attenuated. Figure 2 shows the relationship of $\phi_{\text {out }}$ and attenuation. For $\phi_{\text {out }} \lesssim \phi_{\text {out|peak }}$ the bending is not significant; a deflected beam will not be distinguishable from a direct beam. For $\phi_{\text {out }} \gtrsim \phi_{\text {out|peak }}$, beams that are very strongly deflected are too attenuated to be detectable. Therefore, we have a $\phi_{\text {out } \mid \text { max }}$, a cutoff for $\phi_{\text {out }}$ beyond which attenuation reduces beam intensity below telescope sensitivity. We define $\delta=\phi_{\text {out } \mid \text { max }}-\phi_{\text {out|peak }}$ as the range of $\phi_{\text {out }}$ for which the deflection is significant, while the deflected beam is still detectable. If the Earth is within this range, the strongly deflected beam will be detectable.

The determination of the probability of detection then involves two steps: calculating the probability of the pulsar beams being deflected to the region of detectability, and calculating how often the Earth will lie in this region. This was achieved in Paper III for non-rotating black holes and it was found that with current pulsar population models near the Galactic center, there is a good chance of detection by a multi-year program with future telescopes. Our primary aim in the current paper is to determine whether these estimates change significantly by considering the spin of the SMBH in Sgr A*. The first step in this direction will be to find a version of Figure 2 that applies to a spinning $\mathrm{SMBH}$.

\section{EXPECTED EFFECTS OF SMBH SPIN}

The nature of the spin effects on the $F$ function of Equation (1) are clearest for photon trajectories in the equatorial plane. Figure 1 shows the qualitative features of such trajectories in either the Kerr geometry or the Schwarzschild geometry (for which any plane can be considered the equatorial plane). The deflection is minimum for photons emitted in the radially outward direction (i.e., for $\phi_{\text {in }}=0$ ). As $\phi_{\text {in }}$ increases, photons get closer and closer to the black hole and deflection increases, as is evident in Figure 1. All photons straying too close fall into the black hole. We thus identify an orbit around the black hole with the characteristic that a photon traveling along this orbit circles the black hole forever, so that $\phi_{\text {out }}$ effectively approaches

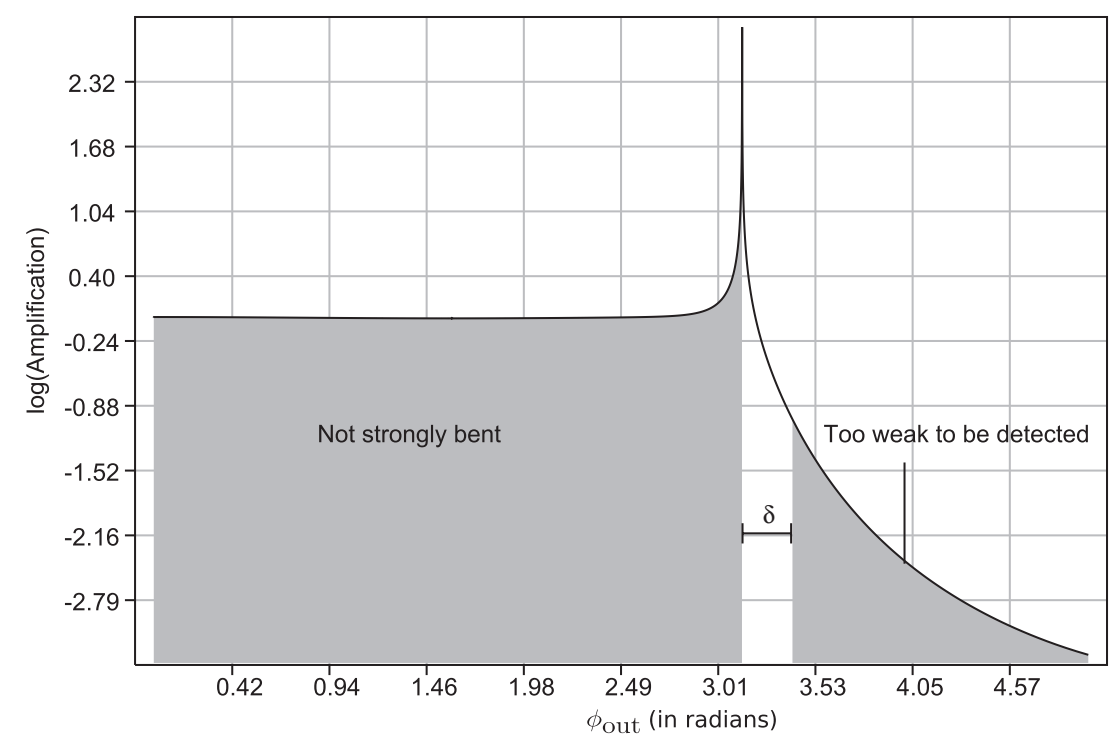

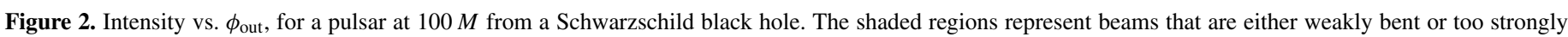
attenuated. 


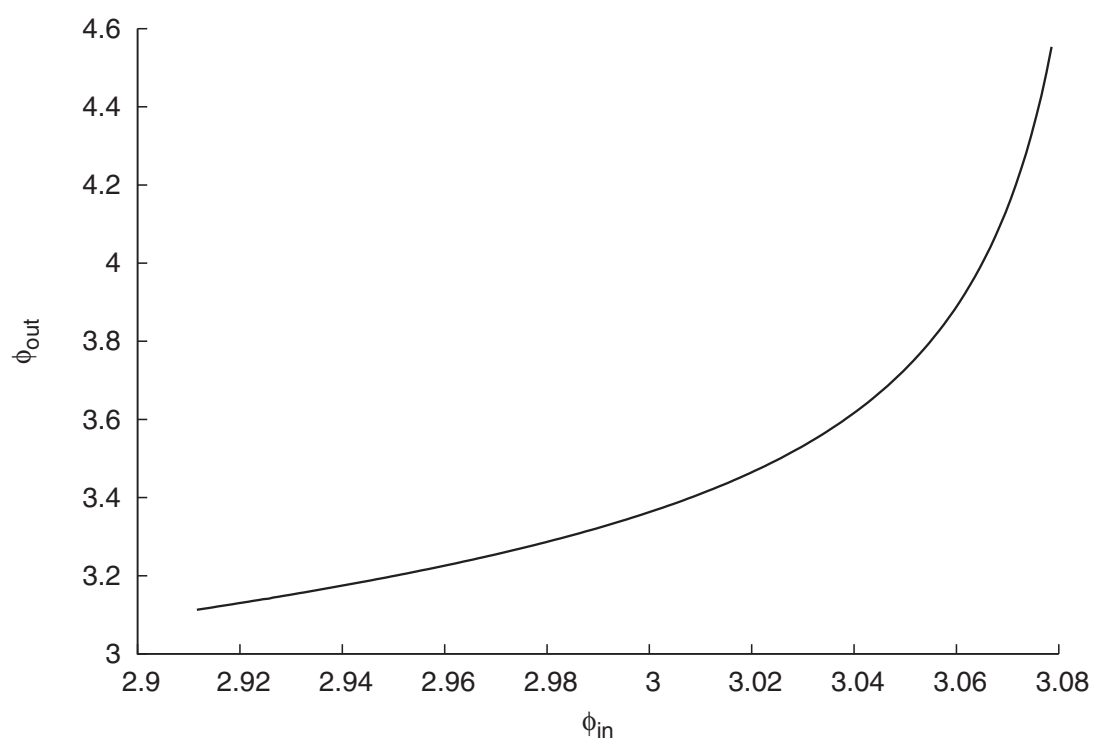

Figure 3. Relation of $\phi_{\text {out }}$ and $\phi_{\text {in }}$ for photons emitted at $r_{0}=100 M$ from a Schwarzschild black hole.

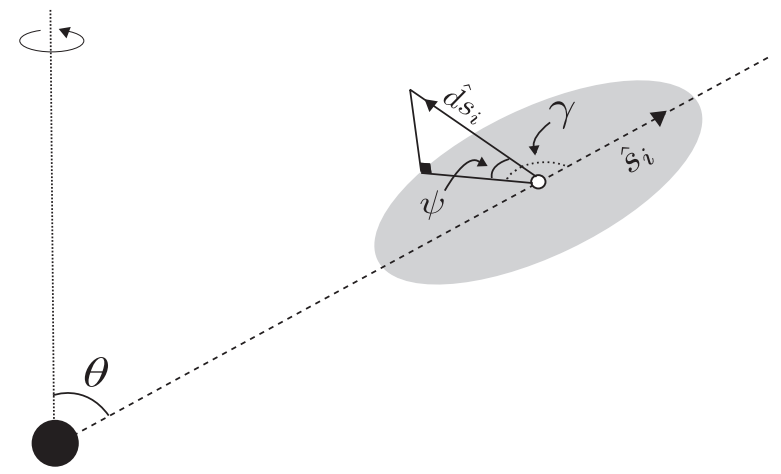

Figure 4. Geometry of a pulsar-Kerr system. The shaded ellipse denotes the plane defined by the pulsar orbital plane and the SMBH. $\psi$ is the angle between the photon direction $\hat{d s_{i}}$ and the projection of $\hat{d s_{i}}$ on the ellipse. $\gamma$ is the angle between this projection and the radially outward direction.

infinity. We use the term unstable circular orbit (UCO) to refer to this orbit and denote the emission angle of a photon traversing this critical trajectory as $\phi_{\text {in } \mid \infty}$. For a Schwarzschild black hole, Figure 3 plots the relationship between $\phi_{\text {out }}$ and $\phi_{\text {in }}$ up to $\phi_{\text {in }} \sim \phi_{\text {in } \mid \infty}$. For larger values of $\phi_{\text {in }}, \phi_{\text {out }}$ is undefined since these trajectories end inside the black hole.

For photons outside a Schwarzschild hole, it is known that the UCO lies at $r=3 \mathrm{M}$. In the case of a spinning black hole, the size of the UCO depends on the relative direction of the angular momentum of the photon and the black hole. We observe that for prograde photons, the size of the UCO decreases as the black hole spin increases whereas for retrograde photons the UCO increases with the black hole spin. A smaller UCO implies $\phi_{\text {in } \mid \infty}$ for a prograde beam outside a spinning black hole will be larger than for a non-spinning black hole. Similarly, a larger UCO for retrograde beams means $\phi_{\text {in } \mid \infty}$ will be smaller than the non-spinning case.

\section{CALCULATION OF PROBABILITY}

The basic geometry outside a Kerr SMBH is illustrated in Figure 4. We will use spherical coordinates $\{r, \theta, \phi\}$ centered at the black hole. In the strong field region, these will be the spatial coordinates of the Boyer-Lindquist system. Since the pulsars of interest are all very far from the SMBH compared to distance $M$, we can consider the spatial geometry to be flat in the neighborhood of the pulsar. In the equations below, the position of the pulsar is specified by $r_{i}$, and $\theta_{i}$ is the angle the pulsar makes with the black hole spin axis at the time of emission. The angles $\gamma$ (the planar angle) and $\psi$ (the axial angle), describing the direction of photon emission are defined as

$$
\begin{gathered}
\cos \gamma=\hat{s_{i}} \cdot \hat{p}\left(\boldsymbol{d} s_{\boldsymbol{i}}\right) \\
\cos \psi=\hat{d s_{i}} \cdot \hat{p}\left(\boldsymbol{d} s_{i}\right)
\end{gathered}
$$

where $\hat{s_{i}}$ is the unit vector in the radially outward direction at the pulsar and $\hat{d s_{i}}$ is a unit vector denoting the initial direction of the emitted photon. The vector $\hat{p}\left(\boldsymbol{d} s_{i}\right)$ denotes the projection of $\hat{d s_{i}}$ in the orbital plane. Four integrals of the equations of motion of a photon in the Kerr geometry can be found. In Boyer-Lindquist coordinates, these are (Misner et al. 1973)

$$
\begin{gathered}
\rho^{2} d \theta / d \lambda=\sqrt{\Theta}, \\
\rho^{2} d r / d \lambda=\sqrt{R} \\
\rho^{2} d \phi / d \lambda=-\left(a E-L_{z} / \sin ^{2} \theta\right)+(a / \Delta) P, \\
\rho^{2} d t / d \lambda=-a\left(a E \sin ^{2} \theta-L_{z}\right)+\left(r^{2}+a^{2}\right) \Delta^{-1} P,
\end{gathered}
$$

where

$$
\begin{gathered}
\Delta=r^{2}-2 M r+a^{2}, \\
\rho^{2}=r^{2}+a^{2} \cos ^{2} \theta, \\
\Theta=\mathcal{Q}-\cos ^{2} \theta\left[-(a E)^{2}+L_{z}^{2} / \sin ^{2} \theta\right], \\
P=E\left(r^{2}+a^{2}\right)-L_{z} a, \\
R=P^{2}-\Delta\left[\left(L_{z}-a E\right)^{2}+\mathcal{Q}\right] .
\end{gathered}
$$

Here, $r, \theta$, and $\phi$ are the (Boyer-Lindquist) coordinates of the photon at different values of affine parameter $\lambda$, and $a=J / M$ is the spin parameter of the hole, where $J$ is the angular momentum 


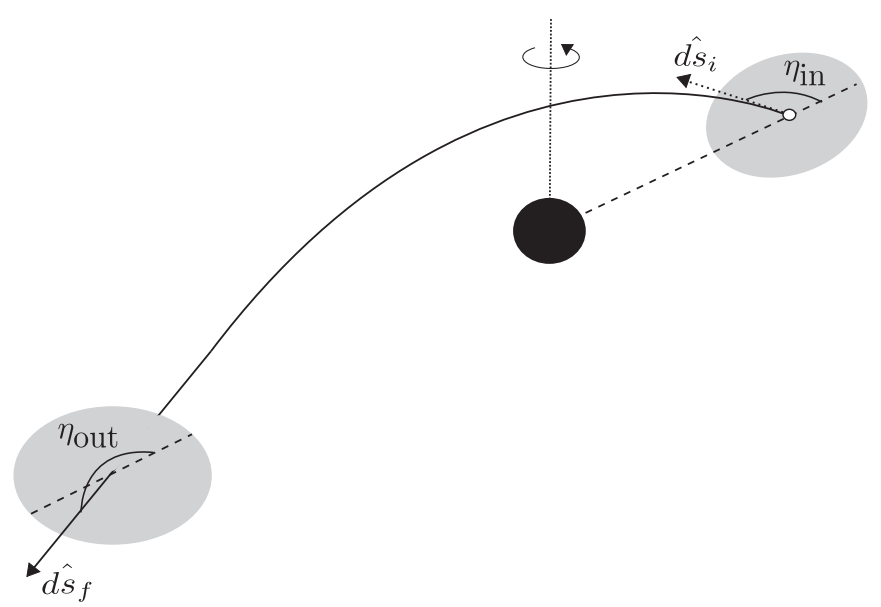

Figure 5. Definitions of $\eta_{\text {in }}$ and $\eta_{\text {out }}$ for a photon trajectory around a spinning black hole.

of the SMBH. The parameters $L_{z}, E$, and $\mathcal{Q}$ (Carter's constant) are the constants of motion.

Our approach will be to initialize $a, r_{i}$, and $\theta_{i}$. We will then use a public FORTRAN code developed by Dexter \& Agol (2009) to evaluate the trajectory of a photon through the strong field region. Along with $a, r_{i}$, and $\theta_{i}$, the code takes as input the three constants of motion and the final radial coordinates and generates final polar coordinates of the photon as output. The final radial coordinates are chosen to be large enough so that for larger radii, there is no significant change in final polar coordinates. The conversion from our geometric parameters to the constants of motion is outlined in the Appendix.

To calculate the attenuation for any given beam, we construct a circular grid of photons closely arrayed around a central photon. Using the Dexter \& Agol code we then calculate the final positions of the central photon and of each photon on the grid. The ratio of the initial and final cross-sectional areas of the grid of photons then gives the attenuation, indicating the change in intensity of the beam. Finally we check that the initial grid is sufficiently small that changing to a smaller cross-section does not give a substantially different result for the attenuation ratio.

\section{RESULTS: OBSERVABILITY OF BEAMS}

To aid us in comparing the pulsar-Kerr black hole system with the pulsar-Schwarzschild black hole system we introduce another set of angles, $\eta_{\text {in }}$ and $\eta_{\text {out }}$, illustrated in Figure 5 and defined as

$$
\begin{gathered}
\cos \eta_{\text {in }}=\hat{s_{i}} \cdot \hat{d s_{i}}=\cos \gamma \cos \psi \\
\cos \eta_{\text {out }}=\hat{s_{i}} \cdot d \hat{s}_{f}
\end{gathered}
$$

where $\hat{s}_{i}$ and $\hat{d s_{i}}$ are as defined in Section 4, and where $d \hat{s}_{f}$ is the spatial unit vector in the direction of the final motion of the photon far from the pulsar-SMBH system. The interpretation of $\eta_{\text {in }}$ in terms of geometric parameters $\gamma$ and $\psi$ comes from their definitions in Figure 4 and Equation (2). Far from the strong field region, $\hat{d}_{f}$ and $\hat{s_{f}}$ point in the same direction. Then, using Equation (A1) from the Appendix for $\hat{s}_{i}$ and $\hat{s}_{f}$ and setting $\phi_{i}=$ 0 for simplicity, the value of $\eta_{\text {out }}$ can be shown to be given by

$$
\cos \eta_{\text {out }}=\cos \theta_{i} \cos \theta_{f}+\sin \theta_{i} \sin \theta_{f} \cos \phi_{f} .
$$

It is expected that the effects of spin of the black hole on the deflection of photons will be most prominent in the equatorial

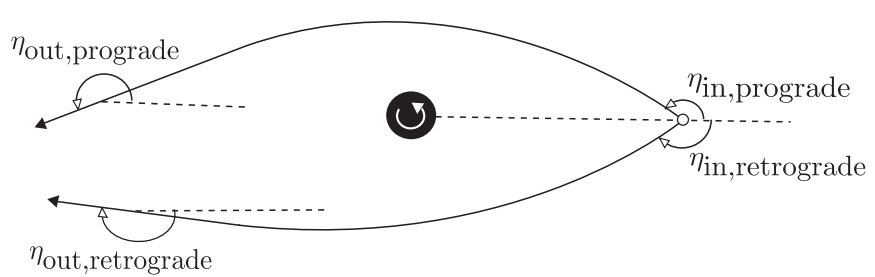

Figure 6. Prograde and retrograde $\eta$ in the equatorial plane of a Kerr black hole.
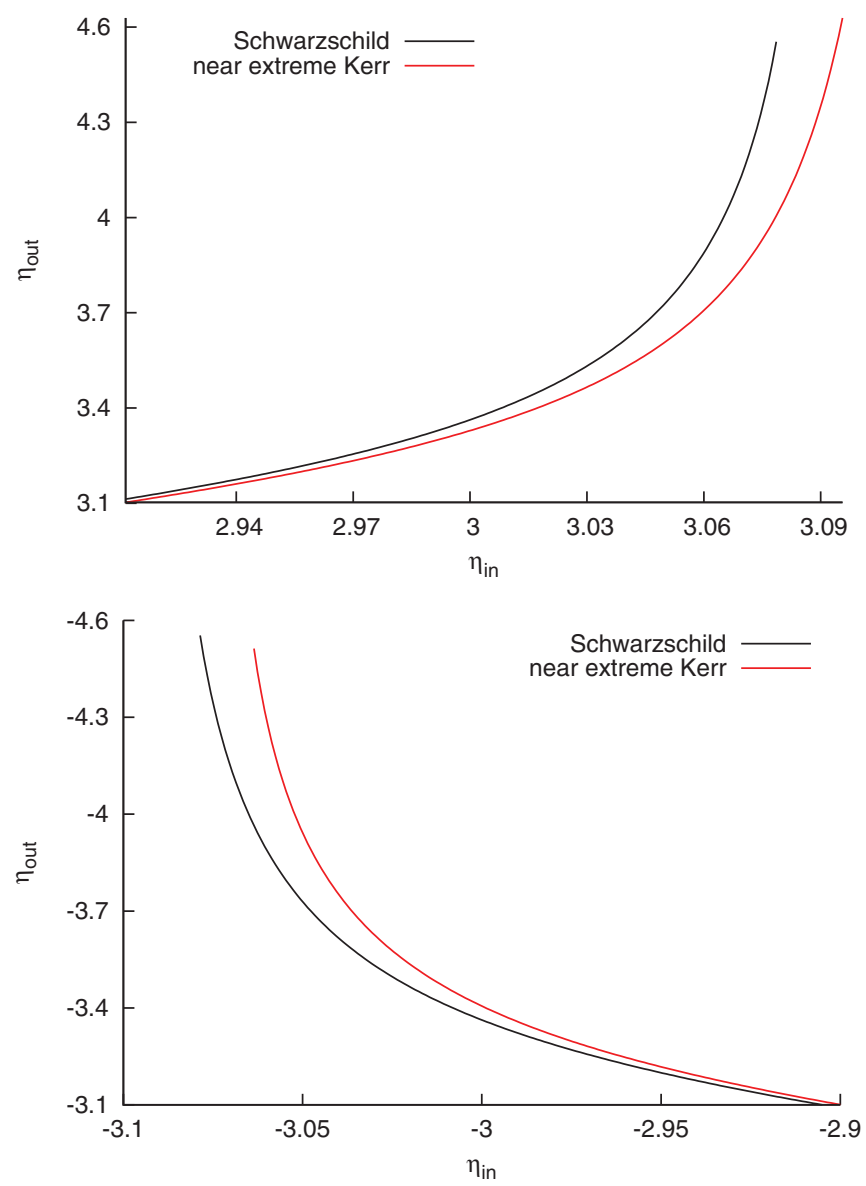

Figure 7. Relationship of $\eta_{\text {out }}$ and $\eta_{\text {in }}$. The top figure shows prograde beams emitted in the equatorial plane of an $a=0.99 \mathrm{SMBH}$ (red) and Schwarzschild SMBH (black) by a pulsar orbiting at a distance of $100 \mathrm{M}$; the bottom figure shows the same comparison for retrograde beams.

(A color version of this figure is available in the online journal.)

plane. As a first step in our investigation of spin effects, we will therefore compute attenuation curves for equatorial Kerr photons similar to the curves in Equation (2). We will assume that the pulsar and the photon trajectories lie in the equatorial plane of the SMBH. (In terms of our geometrical parameters, this corresponds to $\theta_{i}=\pi / 2$ and $\psi=0$.) Figure 6 shows the $\eta$ angles for this simple scenario. The definition of the $\eta$ 's in this scenario is similar to the $\phi$ 's from the Schwarzschild case (Figure 1), and the $\eta$ 's approach the $\phi$ 's as the black hole spin approaches zero.

Figure 7 plots $\eta_{\text {out }}$ as a function of $\eta_{\text {in }}$ for prograde and retrograde beams emitted by a pulsar at a distance of $100 \mathrm{M}$ from an extreme $(a=0.99)$ Kerr SMBH and compares them with the curves for a non-rotating SMBH. There is a late divergence of $\eta_{\text {out }}$ for prograde photons in the near extreme Kerr SMBH case compared to the non-rotating case. This implies a larger $\eta_{\text {in } \mid \infty}$ for prograde Kerr beams than prograde Schwarzschild 

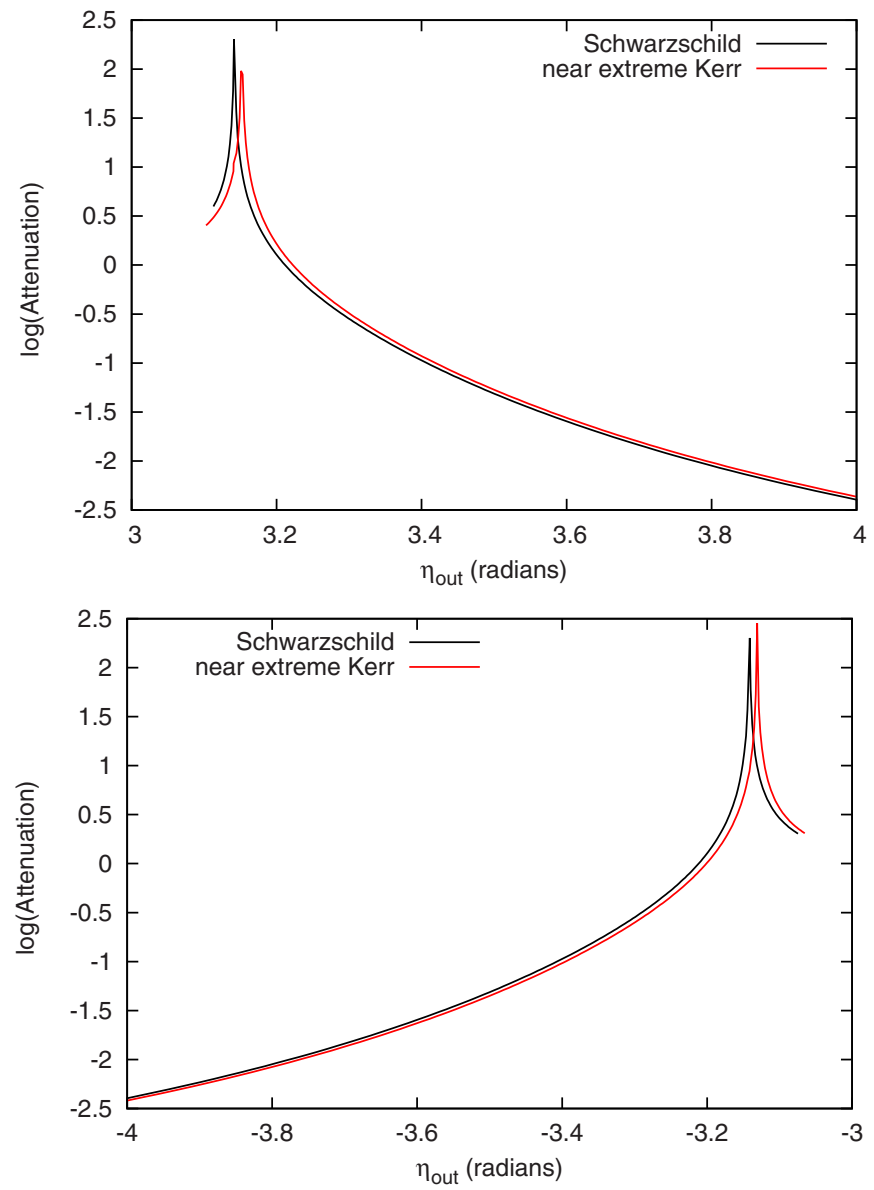

Figure 8. Attenuation of pulse intensity as a function of $\eta_{\text {out }}$ for the same models as in Figure 7. The top figure shows the comparison of the Schwarzschild curve with the prograde case; the bottom show the comparison with the retrograde case.

(A color version of this figure is available in the online journal.)

beams. Similarly, the early divergence of $\eta_{\text {out }}$ for retrograde beams orbiting the near extreme Kerr SMBH implies a smaller $\eta_{\text {in } \mid \infty}$ for retrograde Kerr beams than retrograde Schwarzschild beams, as was observed theoretically in Section 3 .

Attenuation curves are plotted in Figure 8 for the same model as in Figure 7. We notice that spin introduces a very small shift in the attenuation curve. The all-important angle $\delta$ increases by only $3 \%$ for prograde beams in the near extreme Kerr case compared to the Schwarzschild case, and there is almost exactly the same decrease in $\delta$ for the retrograde case. We also notice that as deflection increases the curves are more in agreement, $\eta_{\text {out }}$ at the maximum acceptable attenuation being the same for prograde and retrograde beams in both cases, respectively. Our results show that the deviations get smaller as we go out of the equatorial plane (i.e., $\theta \neq \pi / 2$ or/and $\psi \neq 0$ ). For larger, more relevant pulsar-SMBH distances, the differences become negligible. Thus, spin induces a minimal effect on the attenuation curve of deflected beams, implying that the range of observability of deflected beams calculated for the pulsar-Schwarzschild case remains invariant for a pulsar-nearextreme-Kerr case. The probabilities established in Paper III of an observation with the assumption that the Sgr A* black hole is not rotating are therefore unchanged even if the black hole is spinning very rapidly.

\section{RESULTS: TIMING OF PULSES}

The importance of beam detection lies in the potential to use deflected beams as a probe of the properties of strongly curved spacetime. For such a probe to be useful it should be sensitive to SMBH spin. We have seen that SMBH spin has a negligible effect on beam detectability. It is therefore important to ask whether the effect of spin on pulsar beams is so slight that pulsar timing cannot be used to detect and measure spin.

We aim here only for an order of magnitude demonstration, so we make several simplifying assumptions. First, we assume that the pulsar is in a circular orbit around the SMBH, and that the pulsar beam is confined to the equatorial plane. The timing analysis of TOAs in this case has been thoroughly analyzed in Paper I for a non-spinning SMBH. In that analysis, it was shown that there are two relativistic effects on pulsar TOAs. First there is the timing issue directly connected to the deflection. If the pulsar spins (relative to distant stars) at $\omega$, then beams that arrive at Earth without strong deflection are pulsed at a frequency $\omega$. Strongly deflected beams, however, must be aimed approximately toward the $\mathrm{SMBH}$, and hence, for pulsar orbital angular frequency $\Omega$, are emitted approximately at frequency $\omega \pm \Omega$. The spin of the SMBH will have a small effect on this frequency since it will affect the precise near-SMBH "target" toward which the deflected beam must be aimed. For simplicity we will ignore this small geometric effect and will consider only the second relativistic effect on TOAs - the effect on the time of travel for a photon in a deflected beam.

Since the Earth is nearly stationary relative to the pulsarSMBH system over several pulsar orbits, a pulse emitted from a particular orbital position of the pulsar, and received at the Earth, has a unique $\eta_{\text {out }}$ : the angle of the final direction of the beam with respect to the radially outward direction through the pulsar at the time of emission in a spherical coordinate system centered at the Galactic center (defined in Equation (5), and pictured in Figure 5). The pulse arrival time as a function of $\eta_{\text {out }}$ then represents the variation in TOAs as the pulsar travels through its orbit. $\mathrm{T}\left(\eta_{\text {out }}\right)$, the pulse arrival time, is defined below in terms of the time of travel of photons from the pulsar to the Earth via the strong field region. SMBH spin will affect this time of travel, thus affecting the TOAs. We will use $\mathrm{T}\left(\eta_{\text {out }}\right)$ to investigate the sensitivity of TOAs to SMBH spin.

The change in coordinate time as a pulse traverses the $\mathrm{SMBH}$ and reaches the Earth is given by

$$
\mathcal{T}\left(\eta_{\text {out }} ; R\right)=2 \int_{r_{\min }}^{r_{i}} J(r) d r+\int_{r_{i}}^{R} J(r) d r
$$

where $r_{i}$ is the pulsar-SMBH distance, $R$ is the distance between the SMBH and the Earth, and

$$
J(r)=\frac{d t}{d r}=\frac{P\left(r^{2}+a^{2}\right) \Delta^{-1}+a L_{z}}{\sqrt{R}}
$$

is obtained from the equations of motion of a photon in the Kerr geometry (Equations (3) and (4)). Since the Earth is very far away from the pulsar-SMBH system, we use Equation (7) to find the value of $\mathcal{T}$ at asymptotically large $R$, where relativistic effects are negligible. The pulse arrival time is then defined as

$$
\mathrm{T}\left(\eta_{\text {out }}\right) \equiv \lim _{R \rightarrow \infty}\left[\mathcal{T}\left(\eta_{\text {out }} ; R\right)-\mathcal{T}(\pi ; R)\right]
$$

where we subtract $\mathcal{T}$ at $\eta_{\text {out }}=\pi$ to obtain a finite value for $\mathrm{T}\left(\eta_{\text {out }}\right)$. 

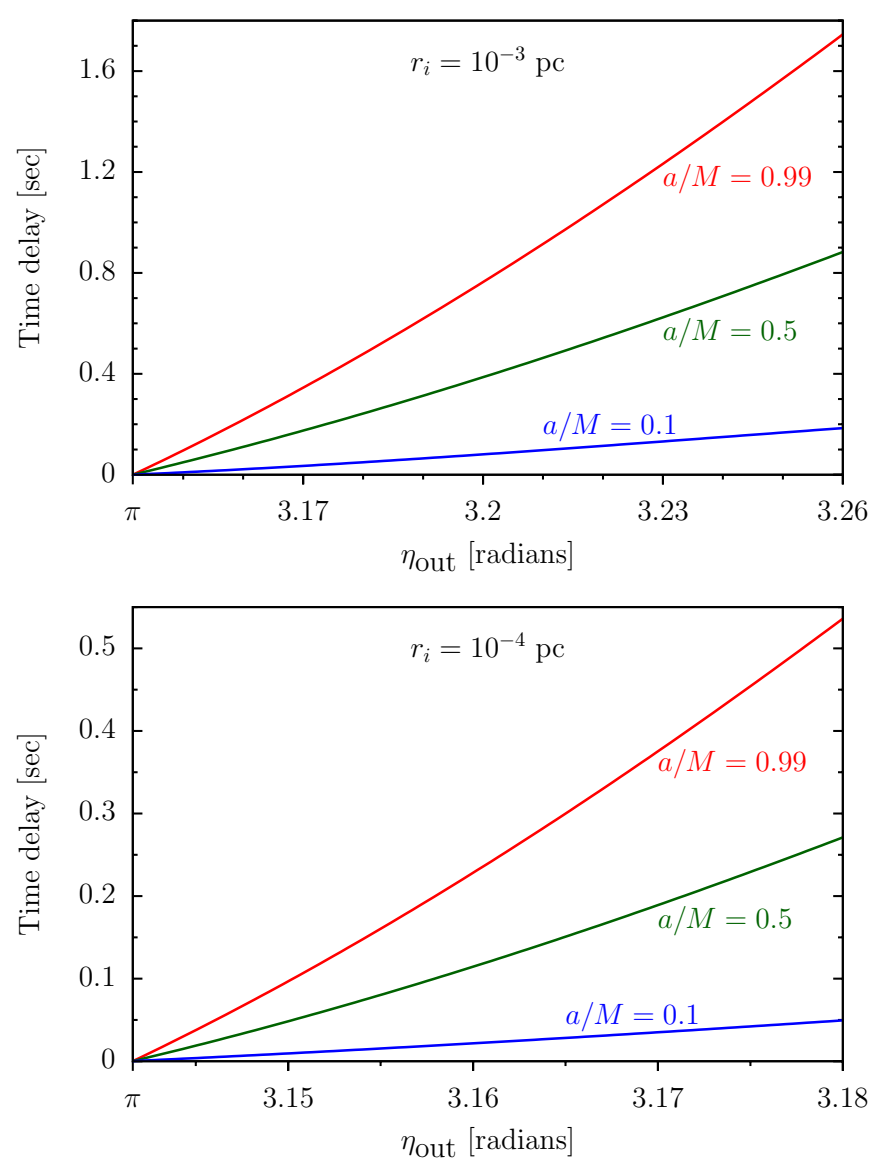

Figure 9. Time delay induced by the SMBH spin as a function of the orbital position of the pulsar for three values of the SMBH spin, labeled by $a / M$. The range of $\eta_{\text {out }}$ is from $\pi$ to $\eta_{\text {out|max }}$. The plots are for pulsar-SMBH distances of $10^{-3} \mathrm{pc}$ and $10^{-4} \mathrm{pc}$ and are labeled accordingly. (See the text for details.)

(A color version of this figure is available in the online journal.)

To illustrate the feasibility of using deflected beams to measure the black hole spin, we calculate $\mathrm{T}\left(\eta_{\text {out }}\right)$ for prograde and retrograde beams. If the $\mathrm{SMBH}$ is not rotating, the prograde and the retrograde beams suffer identical deflection and we find that this Schwarzschild function $\mathrm{T}_{S}\left(\eta_{\text {out }}\right)$ is, of course, the same for the two orientations. If the SMBH is rotating, this symmetry is broken; there will be different values of $\mathrm{T}\left(\eta_{\text {out }}\right)$ for prograde and retrograde orbits. We compare the time delay introduced by SMBH spin, defined as $\mathrm{T}\left(\eta_{\text {out }}\right)-\mathrm{T}_{S}\left(\eta_{\text {out }}\right)$, for prograde and retrograde orientations, respectively. A difference in the time delay for the two orientations can be attributed to the spin of the $\mathrm{SMBH}$.

This measure, and all measures of deflection, depend on the closest approach of the pulsar beam to the SMBH, and that closest-approach distance determines the attenuation of the beams. Since such an analysis will be performed on deflected beams that are detected, the range of $\eta_{\text {out }}$ is restricted by the beam attenuation to be between $\eta_{\text {out|peak }}$, corresponding to peak amplification, and $\eta_{\text {out } \mid \text { max }}$, a cutoff for $\eta_{\text {out }}$ beyond which attenuation is assumed to preclude observation (see Section 2 for details).

For the SMBH at the center of our Galaxy with a mass close to $4.3 \times 10^{6} M_{\odot}$ (Gillessen et al. 2009; Ghez et al. 2008), Figure 9 shows the results of such an analysis. The pulsar-SMBH distance in the two plots is $10^{-3} \mathrm{pc}$ and $10^{-4} \mathrm{pc}$, respectively. The three curves in each plot represent the time delay comparison in the case of a slowly, moderately, and rapidly rotating Kerr $\mathrm{SMBH}$, respectively. Several properties of the spin effects are clear from the plot: at any attenuation, the spin-induced time delay increases with the SMBH spin; the effect is larger for stronger deflection, and the sensitivity to deflection depends on the SMBH spin. The plotted values are the absolute values of the time delays. These values are the same for the prograde and retrograde beams. In the two cases the signs are opposite so the total prograde/retrograde difference is twice the values plotted. We also found that, since these effects originate in the portion of the photon orbit close to the SMBH, the effect of the pulsar$\mathrm{SMBH}$ distance on the time delay pattern is negligible. The only influence of the pulsar-SMBH distance is in determining $\eta_{\text {out|max }}$, which decreases as the distance increases. The pulsars close to the Galactic center are expected to be spinning at rates on the order of $1 \mathrm{~s}$, thus the spin effects would induce phase shifts on the order of one cycle. The conclusion, based on our limited analysis, is that pulsar timing of deflected pulses detected at Earth would be able to extract the spin signatures of the Sgr A* SMBH.

This estimate is of course for an oversimplified picture. To get closer to an accurate prediction, we must consider more general models. A particularly important part of such an analysis would be an effect that, like spin, induces different delays for prograde and retrograde beams such as the eccentricity of the orbit. Preliminary calculations suggest that the pattern of timing effects due to the eccentricity of pulsar orbit is, in fact, easily distinguishable from the pattern introduced by the SMBH spin.

\section{CONCLUSION}

The detection and analysis of pulsar beams strongly deflected by the SMBH in Sgr A* holds promise of providing a tool for probing strongly curved regions of spacetime. An important question has been the probability that such beams are detectable. To remedy a shortcoming of earlier estimates of such probability, we have considered here whether the spin of the Sgr A* SMBH can have a significant effect on detectability. We have found that even for conditions in which spin should have its greatest effects (equatorial photon trajectories, near extreme rotation) the effect of spin on detectability is negligible.

While the effect on detectability is negligible, we have demonstrated that the effect of spin on pulsar arrival times is significant enough that these beams, if detected, can provide us a way of determining the spin of the SMBH. The fact that this determination is well within the precision of pulsar timing suggests that the timing can also determine whether the spacetime near the Galactic center deviates from the Kerr geometry. The motivation for an observational program therefore remains strong.

We gratefully acknowledge support by the National Science Foundation under grants AST0545837 and HRD0734800. We also thank the Center for Gravitational Wave Astronomy, the Center for Advanced Radio Astronomy, and the Department of Physics and Astronomy at the University of Texas at Brownsville.

\section{APPENDIX}

\section{CONSTANTS OF MOTION FROM GEOMETRICAL PARAMETERS}

We work in a spherical coordinate system centered at the black hole. As argued before, the pulsars are far enough from the black hole for us to assume the spacetime is flat there. 
From Figure 4 we then have

$$
\boldsymbol{s}=r \cos \theta \hat{z}+r \sin \theta \cos \phi \hat{x}+r \sin \theta \sin \phi \hat{y},
$$

from which we find

$$
\begin{aligned}
\boldsymbol{d} \boldsymbol{s}_{i}= & \left(\cos \theta_{i} d r-r_{i} \sin \theta_{i} d \theta\right) \hat{z} \\
& +\left(\sin \theta_{i} \cos \phi_{i} d r+r \cos \theta_{i} \cos \phi_{i} d \theta\right. \\
& \left.-r_{i} \sin \theta_{i} \sin \phi_{i} d \phi\right) \hat{x} \\
& +\left(\sin \theta_{i} \sin \phi_{i} d r+r_{i} \cos \theta_{i} \sin \phi_{i} d \theta\right. \\
& \left.+r_{i} \sin \theta_{i} \cos \phi d \phi\right) \hat{y}
\end{aligned}
$$

and

$$
\begin{aligned}
\boldsymbol{p}\left(\boldsymbol{d} \boldsymbol{s}_{i}\right)= & \cos \theta_{i} d r \hat{z}+\left(\sin \theta_{i} \cos \phi_{i} d r-r_{i} \sin \theta_{i} \sin \phi_{i} d \phi\right) \hat{x} \\
& +\left(\sin \theta_{i} \sin \phi_{i} d r+r_{i} \sin \theta_{i} \cos \phi_{i} d \phi\right) \hat{y}
\end{aligned}
$$

where $r_{i}, \theta_{i}$, and $\phi_{i}$ are coordinates of the photon at the time of emission in a spherical coordinate system centered at the black hole. The initial choice of $\phi$ is arbitrary; we set $\phi_{i}=0$ for simplicity. The above equations and Equations (2) give us

$$
\cos \gamma=\frac{\boldsymbol{s}_{i} \cdot \boldsymbol{p}\left(\boldsymbol{d} \boldsymbol{s}_{i}\right)}{\left|\boldsymbol{s}_{i}\right|\left|\boldsymbol{p}\left(\boldsymbol{d} \boldsymbol{s}_{i}\right)\right|}=\frac{d r}{\sqrt{(d r)^{2}+\left(r_{i} \sin \theta_{i} d \phi\right)^{2}}}
$$

so that

$$
\tan \gamma=r_{i} \sin \theta_{i}\left(\frac{d \phi}{d r}\right)_{i} .
$$

From those equations we also have

$$
\begin{aligned}
& \cos \psi=\frac{\boldsymbol{d} \boldsymbol{s}_{i} \cdot \boldsymbol{p}\left(\boldsymbol{d} \boldsymbol{s}_{i}\right)}{\left|\boldsymbol{d} \boldsymbol{s}_{i}\right|\left|\boldsymbol{p}\left(\boldsymbol{d} \boldsymbol{s}_{i}\right)\right|} \\
& =\frac{(d r)^{2}+\left(r_{i} \sin \theta_{i} d \phi\right)^{2}}{\sqrt{(d r)^{2}+\left(r_{i} d \theta\right)^{2}+\left(r_{i} \sin \theta d \phi\right)^{2}} \sqrt{(d r)^{2}+\left(r_{i} \sin \theta_{i} d \phi\right)^{2}}},
\end{aligned}
$$

from which it follows that

$$
\tan \psi=\frac{r_{i} d \theta}{\sqrt{(d r)^{2}+\left(r_{i} \sin \theta_{i} d \phi\right)^{2}}}=\frac{r_{i} d \theta}{\sqrt{(d r)^{2}+(\tan \gamma d r)^{2}}}
$$

and finally

$$
\tan \psi=r_{i} \cos \gamma\left(\frac{d \theta}{d r}\right)_{i} .
$$

By specifying $\gamma, \psi$, and initial values of $r$ and $\theta$, we obtain initial values of $d \phi / d r$ and $d \theta / d r$ which we then use to solve Equations (3) and find the constants of motion.

\section{REFERENCES}

Baganoff, F. K., Maeda, Y., Morris, M., et al. 2003, ApJ, 591, 891

Broderick, A. E., Fish, V. L., Doeleman, S. S., \& Loeb, A. 2009, ApJ, 697, 45

Dexter, J., \& Agol, E. 2009, ApJ, 696, 1616

Doeleman, S. S., Weintroub, J., Rogers, A. E. E., et al. 2008, Natur, 455, 78

Faucher-Gigurè, C.-A., \& Kaspi, V. M. 2006, ApJ, 643, 332

Genzel, R., Schödel, R., Ott, T., et al. 2003, Natur, 425, 934

Ghez, A. M., Salim, S., Weinberg, N. N., et al. 2008, ApJ, 689, 1044

Gillessen, S., Eisenhauer, F., Trippe, S., et al. 2009, ApJ, 692, 1075

Levin, Y. 2006, MNRAS, 374, 515

Liu, S., \& Melia, F. 2002, ApJL, 573, L23

Maness, H., Martins, F., Trippe, S., et al. 2007, ApJ, 669, 1024

Misner, C. W., Thorne, K. S., \& Wheeler, J. A. 1973, Gravitation (San Francisco: Freeman)

Muno, M. P., Pfahl, E., Baganoff, F. K., et al. 2005, ApJL, 622, L113

Nayakshin, S., \& Sunyaev, R. 2005, MNRAS, 364, L23

Pfahl, E., \& Loeb, A. 2004, ApJ, 615, 253

Porquet, D., Predehl, P., Aschenbach, B., et al. 2003, A\&A, 407, L17

Schödel, R., Ott, T., Genzel, R., et al. 2002, Natur, 419, 694

Stovall, K., Creighton, T., Price, R. H., \& Jenet, F. A. 2012, ApJ, 744, 143

Wang, Y., Creighton, T., Price, R. H., \& Jenet, F. A. 2009a, ApJ, 705, 1252

Wang, Y., Jenet, F. A., Creighton, T., \& Price, R. H. 2009b, ApJ, 697, 237 